\title{
Application of Bioremediation to Soil Contaminated by Lubricants Around Railroad Turnouts
}

\author{
Jae-Young Lee ${ }^{\dagger}$, Tae-Soon Kwon**, Young-Min Cho*, Hae-Suk Kang* and Woo-Sung Jung*
}

\begin{abstract}
In this study, the feasibility of using bioremediation to treat lubricant-contaminated soil around railroad turnouts was investigated. Lubricants used during the maintenance of railroad turnouts can drip onto the ground causing soil contamination. In the laboratory experiments, the residual TPH (Total Petroleum Hydrocarbons) concentration in soil gradually decreased after microorganisms degrading the lubricants were added. Generally, the soil around railroad turnouts is covered by a layer of ballasts. In the column experiments that were designed considering field sites, the removal efficiency of TPH was about $11 \%$ after 60 days of cultivation time. In the field experiments, microorganisms were added into the soil periodically, and finally the residual TPH concentrations were reduced to less than $1,700 \mathrm{mg} / \mathrm{kg}$-soil on average. These results indicate that the lubricant in the contaminated soil around railroad turnouts could be efficiently removed through bioremediation method.
\end{abstract}

Keywords : Bioremediation, Lubricants, Railroad turnout, Soil contamination

\section{Introduction}

Soil pollution around railroad turnouts is mainly caused by drops of lubricants that are regularly used during their maintenance [1-3]. Lubricants are composed of molecules that have higher carbon numbers than diesel oil or gasoline, common organic contaminants found in soil [2-3]. Physical and chemical methods have been applied to treat lubricant-contaminated soil, but these methods are generally ineffective and expensive [2-3]. Also, it is difficult to suspend operation of rolling stocks so that the contaminated soil can be treated. Considering the difficulties of these methods, bioremediation method can be an environmentally -friendly and cost-effective alternative [4-6]. J.K. Adesodun and J.S.C. M bagwu (2008) studied the bioremediation of soils containing waste-lubricant oil using animal droppings. Sang-Hwan Lee, et al. (2007) reported the extent of lubricant biodegradation in different nutrient con-

\footnotetext{
$\dagger$ Corresponding author: Railroad Environment Research Development, Korea Railroad Research Institute

E-mail : iyoung@krri.re.kr

* Railroad Environment Research Development, Korea Railroad Research Institute

** Railroad Testing \& Certification Center, Korea Railroad Research Institute
}

ditions, as well as the monitoring process of bioremediation in soil contaminated with lubricants. The purpose of this study was to use bioremediation for the treatment of lubricant-contaminated soil around railroad turnouts. Laboratory, column and field experiments were performed to evaluate the feasibility of using bioremediation.

\section{Materials and Methods}

\subsection{Contaminated soil and microorganisms}

The soil used in this study was collected below the layer of ballasts around railroad turnouts at several stations and depots in Korea. The soil types consisted of sandy-silt. The initial $\mathrm{pH}$ of soil was 6.14 and the water content was $2.8 \mathrm{wt} \%$. Main pollutants in the soil were lubricants, which were shown as TPH (Total Petroleum Hydrocarbons). The initial TPH concentration was about $43,300 \mathrm{mg} / \mathrm{kg}$-soil,

Table 1. Standard Level for the Main Pollutants of Railroad Site According to the Soil Environment Preservation Law in Korea

\begin{tabular}{ccccccc}
\hline Pollutants & $\mathrm{TPH}$ & $\mathrm{Cd}$ & $\mathrm{Cu}$ & $\mathrm{Pb}$ & $\mathrm{Zn}$ & $\mathrm{Ni}$ \\
\hline $\begin{array}{c}\text { Standard level } \\
\text { (mg/kg soil) }\end{array}$ & 2,000 & 60 & 2,000 & 700 & 2,000 & 500 \\
\hline
\end{tabular}


which was much higher value than the $2,000 \mathrm{mg} / \mathrm{kg}$-soil of the standard level designated from the Soil Environment Preservation Law (Korea) as shown in Table 1. Ecoguard CDM (EcoPhile, Korea) with lubricant-degrading microorganisms (mainly Rhodcoccus sp., Pseudomonas sp., Sphingomonas sp.) was used. The medium was Ecoguard MS I (EcoPhile, Korea) which is composed of various nitrogen and phosphate sources such as $\left(\mathrm{NH}_{4}\right)_{2} \mathrm{HPO}_{4}$ and $\mathrm{KH}_{2} \mathrm{PO}_{4}$.

\subsection{Biodegradation experiments}

Bioremediation method on lubricants-contaminated soil around railroad turnouts was tested in laboratory experiments. $500 \mathrm{~g}$ of soil was added to a $3 \mathrm{~L}$ of stainless steel container. The initial concentration of microorganisms was $3 \mathrm{~kg} / \mathrm{m}^{3}$-soil. The medium was added to $350 \mathrm{ppm}$ per $1,000 \mathrm{ppm}$ of TPH with a 100/10/1 C/N/P ratio. In order to enhance the efficiency of lubricant biodegradation through co-oxidation, $0.1 \%$ of kerosene was used additionally. The cultivation temperature was $25^{\circ} \mathrm{C}$ and tilling was done every three day. Water was adjusted twice a week. A control experiment was conducted, in which microorganisms and medium were not added, to identify self-biodegradation of lubricants from the contaminated soil. Soil samples were collected periodically $(0,4,11,18$, and 25 days) to analyze the residual TPH concentration in soil.

Column experiments were conducted to apply bioremediation method in the field. As shown in Fig. 1, the column was cylindrical in shape, and it was $210 \mathrm{~mm} \times$ $500 \mathrm{~mm}(\mathrm{R} \times \mathrm{H})$. The column was filled with $10 \mathrm{~cm}$ of contaminated soil, and a layer of ballasts was added on top in order to emulate the conditions in the railroad site. In the beginning of the experiment, $100 \mathrm{~mL}$ of microorgan-

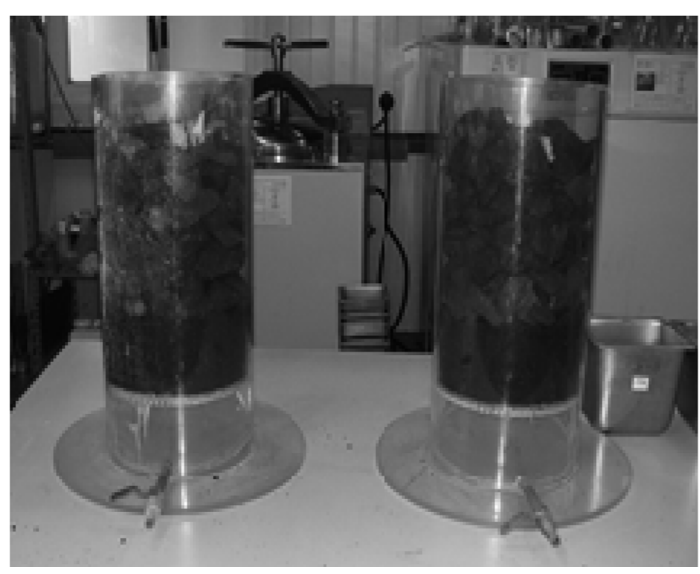

Fig. 1 Photograph of column experiments for the bioremediation of the contaminated soil around railroad turnouts

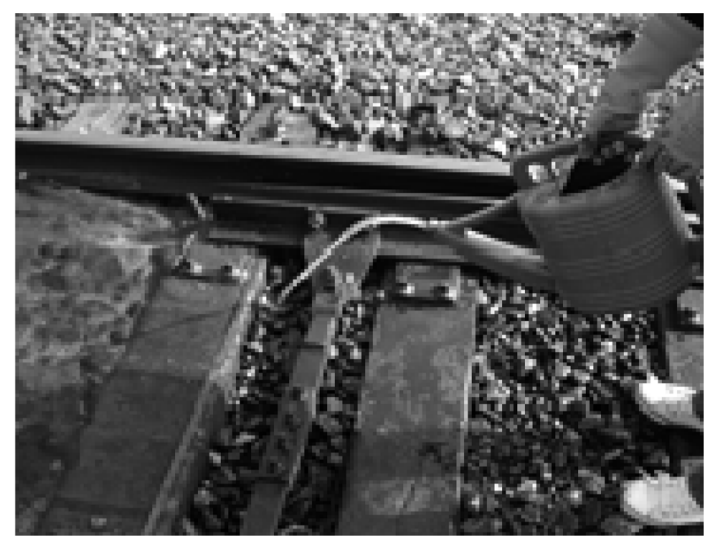

Fig. 2 Photograph of field experiments for the bioremediation of the contaminated soil around railroad turnouts

isms and medium were injected into the samples, and then considering the initial TPH concentration of the soil, $50 \mathrm{~mL}$ of microorganisms were sprinkled into the column every week. As in the laboratory test, a control group, to which microorganisms and medium were not added, was used in the column test. After 60 days of cultivation time, finally the residual TPH concentration in the soil was measured.

Field experiments were carried out at a railroad vehicle office (Korea) from the beginning of April to the end of September in 2008. Fig. 2 shows to inject microorganisms into the contaminated soil around railroad turnouts in the field. Generally, the use of lubricants in the maintenance of the railroad turnouts was ongoing during the field experiments, which could be the main cause of re-contamination in the field. Initial TPH concentrations from the contaminated soil around railroad turnouts were from 8,500 to $10,000 \mathrm{mg} / \mathrm{kg}$-soil. Considering the initial $\mathrm{TPH}$ concentration in soil, $20 \mathrm{~L}$ of microorganisms and $60 \mathrm{~L}$ of medium were spread evenly over the soil around the railroad turnouts at the beginning of the experiment. $20 \mathrm{~L}$ of microorganisms were then added periodically for a 6 month period.

In order to analyze the residual TPH concentration in the soil, the samples from the three designated areas around the railroad turnouts were collected at $0-30 \mathrm{~cm}$ depths before the microorganisms and medium were added repeatedly.

\subsection{Analysis}

Soil samples were dried at $105^{\circ} \mathrm{C}$ for $24 \mathrm{hr}$, and then sieved to less than $2 \mathrm{~mm}$. $100 \mathrm{~mL}$ of dichloromethane (Honeywell Burdick \& Jackson, USA) was mixed with $10 \mathrm{~g}$ of soil using an ultrasonic processor (VC 750, USA) to extract TPH from the soil. After centrifugation, an 
upper phase was injected into GC (Varian 3900, USA) equipped with FID and VH-5 column (30 m length, $0.25 \mathrm{~mm}$ internal diameter and $0.25 \mu \mathrm{m}$ film thickness). All steps were performed under following conditions: initial temperature $45^{\circ} \mathrm{C}$; initial isothermal for $2 \mathrm{~min}$; temperature rate $10^{\circ} \mathrm{C} / \mathrm{min}$; final temperature $310^{\circ} \mathrm{C}$; final isothermal for $25 \mathrm{~min}$; injector temperature $280^{\circ} \mathrm{C}$; detector temperature $300^{\circ} \mathrm{C}$; carrier gas(nitrogen) flow rate $1 \mathrm{ml} / \mathrm{min}$.

\section{Results and Discussions}

\subsection{Laboratory experiments}

As presented in Fig. 3, the residual TPH concentrations of soil in the control group showed almost no change. In the other hand, the residual TPH concentration of soil with lubricant-degrading microorganisms was significantly decreased. After 18 days, the removal efficiency of TPH was about $30 \%$ and the biodegradation rate of TPH was slowed down gradually. The final removal efficiency of TPH was about $34 \%$ and the final TPH concentration in the soil was about $29,000 \mathrm{mg} / \mathrm{kg}$-soil.

\subsection{Column experiments}

Fig. 4 depicts the change of residual TPH concentration in the lubricant-contaminated soil in the column experiments. The addition of microorganisms decreased the residual TPH concentration in soil at a much higher rate than the control group, just like laboratory experiments. After 60 days of total cultivation time, the color of the soil had faded apparently, and the removal efficiency of TPH

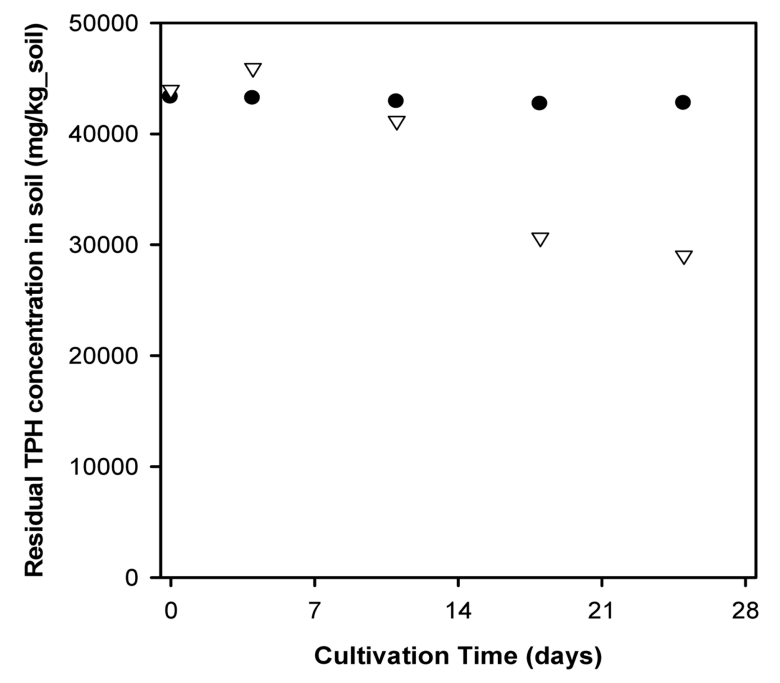

Fig. 3 The change of residual TPH concentration in the lubricants-contaminated soil around railroad turnouts $\mathrm{w}(\nabla)$

/o (๑) microorganisms in the laboratory experiments

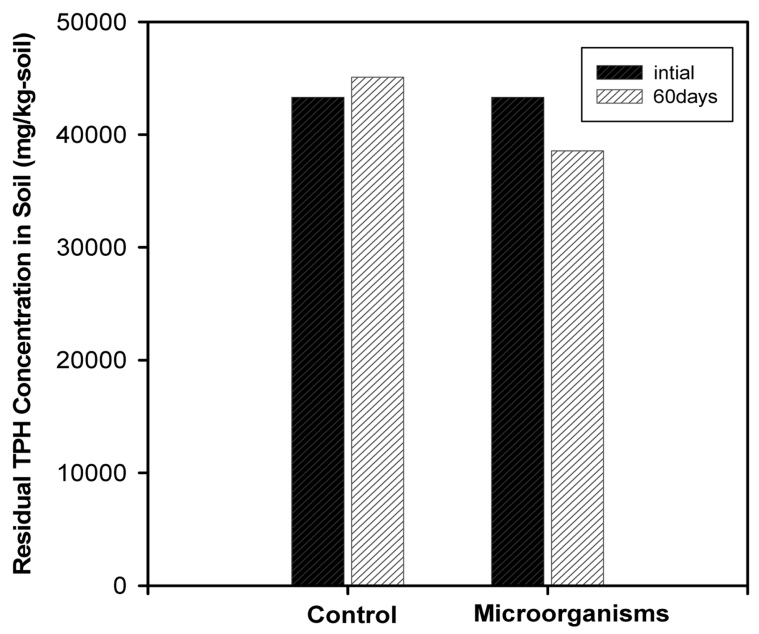

Fig. 4 The change of residual TPH concentration in the lubricant-contaminated soil around railroad turnouts after 60 days in the column experiment

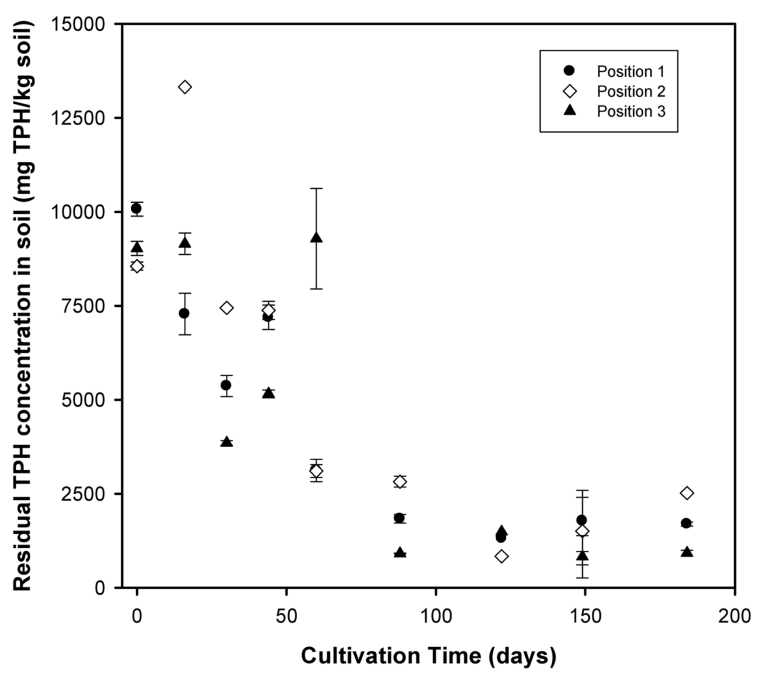

Fig. 5 The change of residual TPH concentration in the lubricant-contaminated soil around railroad turnouts during the month-long field experiment

was about $11 \%$. This result was lower than that of the laboratory experiments. This was due to the fact that tilling could not be done in the column experiment because of the ballasts on top of the soil.

\subsection{Field experiments}

The change of residual TPH concentrations in the soil that occurred during the 6 month in the long field experiment is described in Fig. 3. As microorganisms were added periodically, the residual TPH concentrations in soil gradually decreased. The average removal efficiency of TPH was about $54 \%$ and the final TPH concentrations in 
soil were about $1,700 \mathrm{mg} / \mathrm{kg}$-soil. From these results, it was determined that bioremediation method could be an alternative treatment method for the lubricant-contaminated soil around railroad turnouts.

\section{Conclusions}

Lubricants are continually applied in the maintenance of railroad turnouts to ensure optimal railroad safety and operation. Soil contamination around railroad turnouts is results from the drops of lubricants. Bioremediation is one effective method for cleaning up the soil contaminated by lubricants around railroad turnouts. Most of lubricant-contaminated soil samples showed initially high TPH concentrations. When microorganisms that degrade lubricants were added into the contaminated soil, the residual TPH concentrations were reduced. At the field site, more than $50 \%$ of the lubricants in the soil were eliminated over a 6 month period by periodic addition of microorganisms. In the future, the bioremediation method can be applied to improve soil contamination around railroad turnouts.

\section{Acknowledgements}

The authors gratefully acknowledge the financial support from MKE (Ministry of Knowledge Economics).

\section{References}

1. Oa S.-W. and Lee T.-G. (2009). "Investigation of soil pollution status for railroad depot," Journal of the Korean Society for Railway, Vol. 12, No. 5, pp. 788-792.

2. Park, S.-W., Lee, J.-Y., Yang, J.-S., Kim, K.-J. and Baek K. (2009). "Electrokinetic remediation of contaminated soil with waste-lubricant oils and zinc," Journal of Hazardous Materials, Vol. 169, pp. 1168-1172.

3. Park, S.-W., Lee, J.-Y., Kim, K.-J., Yang, J.-S. and Baek, K. (2010). "Alkaline enhanced-separation of waste lubricant oils from railway contaminated soil," Separation and Science Technology, Vol. 45, No. 12-13, pp. 1988-1993.

4. Adesodun, J. K. and Mbagwu, J.S.C. (2008). "Distribution of heavy metals and hydrocarbon contents in an alfisol contaminated with waste-lubricating oil amended with organic wastes," Bioresource Technology, Vol. 99, pp. 3195-3204.

5. Adesodun, J. K. and Mbagwu, J.S.C. (2008). "Biodegradation of waste-lubricating petroleum oil in a tropical alfisol as mediated by animal droppings," Bioresource Technology, Vol. 99, pp. 5659-5665.

6. Lee, S.-H., Lee, S.H., Kim, D.-Y. and Kim, J.-G. (2007). "Degradation characteristics of waste lubricants under different nutrient conditions," Journal of Hazardous Materials, Vol. 143, pp. 65-72.

Received(February 10, 2011), Revised(February 28, 2011), Accepted(March 15, 2011) 